\title{
NASA'S PLANETARY GEOLOGIC MAPPING PROGRAM: OVERVIEW
}

\author{
D. A. Williams
}

School of Earth \& Space Exploration, Arizona State University, Tempe, Arizona, USA - david.williams@asu.edu

\author{
Commission IV, WG IV/8
}

KEY WORDS: Planetary Cartography, Geologic Mapping, NASA Missions

\begin{abstract}
:
NASA's Planetary Science Division supports the geologic mapping of planetary surfaces through a distinct organizational structure and a series of research and analysis (R\&A) funding programs. Cartography and geologic mapping issues for NASA's planetary science programs are overseen by the Mapping and Planetary Spatial Infrastructure Team (MAPSIT), which is an assessment group for cartography similar to the Mars Exploration Program Assessment Group (MEPAG) for Mars exploration. MAPSIT's Steering Committee includes specialists in geological mapping, who make up the Geologic Mapping Subcommittee (GEMS). I am the GEMS Chair, and with a group of 3-4 community mappers we advise the U.S. Geological Survey Planetary Geologic Mapping Coordinator (Dr. James Skinner) and develop policy and procedures to aid the planetary geologic mapping community. GEMS meets twice a year, at the Annual Lunar and Planetary Science Conference in March, and at the Annual Planetary Mappers' Meeting in June (attendance is required by all NASA-funded geologic mappers). Funding programs under NASA's current R\&A structure to propose geological mapping projects include Mars Data Analysis (Mars), Lunar Data Analysis (Moon), Discovery Data Analysis (Mercury, Vesta, Ceres), Cassini Data Analysis (Saturn moons), Solar System Workings (Venus or Jupiter moons), and the Planetary Data Archiving, Restoration, and Tools (PDART) program. Current NASA policy requires all funded geologic mapping projects to be done digitally using Geographic Information Systems (GIS) software. In this presentation we will discuss details on how geologic mapping is done consistent with current NASA policy and USGS guidelines.
\end{abstract}

\section{INTRODUCTION}

NASA's Planetary Science Division supports the geologic mapping of planetary surfaces through a distinct organizational structure and a series of research and analysis (R\&A) funding programs as part of its overall Cartography Program. This presentation reviews the purpose of geologic mapping, organization structure to support PIs with map production via the USGS Astrogeology Science Center (ASC), funding programs to propose mapping projects, and the overall approach to planetary mapping.

\subsection{Why Planetary Geologic Mapping?}

Geologic mapping is an investigative process used to understand the evolution of the terrestrial planets and satellites. The goal of geologic mapping is to place observations of surface features into their stratigraphic context in order to develop generalized geologic timescales that provide a sequence of events for the evolution of planetary surfaces. For example, the lunar geologic timescale was developed by correlation of absolute ages obtained from Apollo samples with relative ages obtained from planetary mapping using telescopic \& Lunar Orbiter photographs. For other planets and satellites, understanding their geologic evolution requires mapping of relative stratigraphy and estimates of ages derived from impact crater populations.

The advantage of geologic mapping relative to photogeologic analyses alone is that it reduces the complexity of heterogeneous planetary surfaces into comprehensible proportions. Discrete material units are defined and characterized according to specific physical attributes that are related to the geologic processes that produced them. These attributes include albedo, texture, surface morphology, topography, and color or compositional information if available. Along with visible structural features, the distributions of these units are then mapped to identify the relative roles of impact cratering, volcanic, tectonic and gradational processes that shape planetary surfaces. The stratigraphic techniques originally developed for the early lunar studies are now the basis for the study of other rocky and icy bodies in the solar system (Wilhelms, 1990).

Planetary geologic mapping has been applied to those terrestrial planets, outer planet satellites, and small bodies (dwarf planets, asteroids and comets) for which adequate image coverage is available. The interpretation of planetary surfaces is done within the context of the exogenic \& endogenic geologic processes that resulted in their present state. These two broad classes of geologic processes have had various relative roles on different planets and satellites, which can be assessed from stratigraphic studies. It should be emphasized that geologic maps are tools that have aided in the understanding of the processes that produced morphologically-distinct materials observed throughout the Solar System, and that (in general) chemical \& physical analyses of a given site on a planetary body can be investigated far better if the geologic setting of the site is known first. This is a fundamental concept in terrestrial geology, but it is a concept that has found slow acceptance in the exploration of other planets \& satellites in the Solar System.

\section{ORGANIZATION}

Cartography and geologic mapping issues for NASA's planetary science programs are overseen by the Mapping and Planetary Spatial Infrastructure Team (MAPSIT), which is an assessment group for cartography similar to the Mars Exploration Program Assessment Group (MEPAG) for Mars exploration. MAPSIT's Steering Committee includes specialists in geological mapping, who make up the Geologic Mapping Subcommittee (GEMS). David A. Williams is the current GEMS Chair, and with a group of 3-4 community mappers we advise the U.S. Geological Survey Planetary Geologic Mapping Coordinator (James A. Skinner) and develop policy and procedures to aid the planetary geologic mapping community. 
GEMS meets twice a year, at the Annual Lunar and Planetary Science Conference in March, and at the Annual Planetary Mappers' Meeting in June (attendance is required by all NASAfunded geologic mappers).

\subsection{Funding}

Funding programs under NASA's current Research \& Analysis structure to which one can propose geological mapping projects include Mars Data Analysis (Mars), Lunar Data Analysis (Moon), Discovery Data Analysis (Mercury, Vesta, Ceres), Cassini Data Analysis (Saturn moons), Solar System Workings (Venus or Jupiter moons, or comparative planetology maps), and the Planetary Data Archiving, Restoration, and Tools (PDART) program. When writing mapping proposals, PIs should communicate with JAS prior to submission to consult on basemap products, map scales, and planned support from the USGS for the project. Details are provided in the Planetary Science Overview (Section C.1) in the annual Research Opportunities in Space and Earth Sciences (ROSES) Announcement of Opportunity (AO).

\subsection{Mapping Approach}

Guidelines for making geologic maps are given in the Planetary Mappers Handbook, which is a downloadable PDF available at the United States Geological Survey Astrogeology Science Center (USGS-ASC) website (Tanaka et al., 2010). Current NASA policy requires all funded geologic mapping projects to be done digitally using Geographic Information Systems (GIS) software. ArcGISTM software by ESRI is preferred by the USGS-ASC, which supports funded mappers through their own GIS laboratory and through technical support. Principal Investigators (PI) should have their own mapping computers and access to ArcGIS ${ }^{\mathrm{TM}}$ software at their home institutions prior to start of the project. It is recommended that mappers obtain basic ArcMap ${ }^{\mathrm{TM}}$ training through ESRI online classes or other means before the start of the project.

Once a mapping project is funded, the USGS-ASC will provide the PI with a digital ArcGISTM project including preferred basemap, possibly some supplementary data sets, and layers for mapping linear features (lines), point features (points), geologic contacts (lines), surface features (polygons), and map units (polygons). Mappers proceed to produce the digital map in GIS as well as the map text, reporting results or discussing problems at the annual Planetary Mappers Meeting. At any time the staff of the USGS-ASC is available to answer questions or help diagnose problems during the mapping process.

Once a draft map and text is completed, it is submitted to the USGS for review. James Skinner will check it for accuracy with Handbook guidelines, and then the map will undergo peer review by two mappers, as well as a map coordinator review. After revision for any reviewer comments, the map will again be checked by JAS and then sent to USGS Publications for production. In the past, this process from start to finish typically lasted 5 years. However, with the advent of digital map production and the refinement of procedures, it is hoped this timeline can be reduced to 3-4 years total.

\section{CONCLUSIONS}

NASA has supported the geological mapping of planetary surfaces since the 1960s, working in conjunction with the U.S. Geological Survey through a variety of organizational structures. Since the 1960s NASA and the USGS have overseen geological mapping campaigns for the Moon, Mars, Venus, and Mercury, and has supported mapping of the Jovian satellites, asteroid Vesta, and the dwarf planet Ceres, using data returned by a variety of robotic missions. MAPSIT and GEMS are currently working with NASA to streamline procedures for planetary geological mapping in the digital age, so that the planetary mapping community has a robust infrastructure and resources to continue mapping of planetary objects to support future science and exploration.

Please contact David Williams (David.Williams@asu) or James Skinner (jskinner@usgs.gov) for questions about the mapping program.

\section{REFERENCES}

Tanaka, K.L., et al., 2010. Planetary Geologic Mapping Handbook, available http://astrogeology.usgs.gov/Projects/PlanetaryMapping/

Wilhelms, D.E., 1990. Geologic mapping, in Planetary Mapping, edited by R. Greeley and R.M. Batson, Cambridge Univ. Press, New York, pp. 208-260. 